\title{
Adherence To Respiratory And Nonrespiratory Medication In Patients With COPD: Results Of The German COSYCONET Cohort
}

This article was published in the following Dove Press journal: Patient Preference and Adherence

\begin{abstract}
Norbert Königsdorfer,' Rudolf A Jörres, (D)' Sandra Söhler, (D) ${ }^{2}$ Tobias Welte, ${ }^{3}$ Jürgen Behr, ${ }^{4}$ Joachim H Ficker, (iD) ${ }^{5}$ Robert Bals, ${ }^{6}$ Henrik Watz, 7 Johanna I Lutter, (iD) ${ }^{8}$ Tanja Lucke, (D) ' Frank Biertz, (iD) 9 Peter Alter, (iD) ${ }^{10}$ Claus $F$ Vogelmeier, ${ }^{10}$ Kathrin Kahnert ${ }^{4}$

'Institute and Outpatient Clinic for Occupational, Social and Environmental Medicine, Comprehensive Pneumology Center Munich (CPC-M), Ludwig-Maximilians-Universität München, Munich 80336, Germany; ${ }^{2}$ ASCONET Study Coordination Office, University of Marburg, Marburg 35043, Germany;

${ }^{3}$ Department of Pneumology, Hannover Medical School, Hannover 30625, Germany; ${ }^{4}$ Department of Internal Medicine V, University of Munich, Comprehensive Pneumology Center, Member of the German Center for Lung Research, Munich 80336, Germany; ${ }^{5}$ Department of Respiratory Medicine, Allergology and Sleep Medicine, General Hospital Nuernberg, Paracelsus Medical University, Nuernberg, Germany; ${ }^{6}$ Department of Internal Medicine V, Pneumology, Allergology, Respiratory Intensive Care Medicine, Saarland University Hospital, Homburg 66424, Germany;

${ }^{7}$ Pulmonary Research Institute at LungenClinic

Grosshansdorf, Airway Research Center North, Member of the German Center for Lung Research, Grosshansdorf 22927, Germany; ${ }^{8}$ Institute of Health Economics and Health Care Management, Helmholtz Zentrum München GmbH - German Research Center for Environmental Health, Comprehensive Pneumology Center Munich (CPC-M), Munich 85764, Germany; ${ }^{9}$ Institute for Biostatistics, Hannover Medical School, Hannover 30625,

Germany; ${ }^{10}$ Department of Medicine, Pulmonary and Critical Care Medicine, University Medical

Center Giessen and Marburg, Philipps-University Marburg, Marburg 35043, Germany
\end{abstract}

Correspondence: Kathrin Kahnert Department of Internal Medicine V, University of Munich, Comprehensive Pneumology Center,

Member of the German Center for Lung Research, Ziemssenstr. I, Munich 80336, Germany

Tel +498944002590

Email Kathrin.Kahnert@med.uni-muenchen.de
Background: Adherence to COPD medication is often considered to be lower than in other chronic diseases. In view of the frequent comorbidities of COPD, the economic impact of nonadherence and the potential for adverse effects, a direct comparison between the adherence to respiratory and nonrespiratory medication in the same patients seems of particular interest.

Objectives: We aimed to investigate the intake of respiratory and nonrespiratory medication in the same patients with COPD and frequent comorbidities.

Method: Within the COPD cohort COSYCONET, we contacted 1042 patients, mailing them a list with all medication regarding all their diseases, asking for regular, irregular and nonintake.

Results: Valid responses were obtained in 707 patients covering a wide spectrum of drugs. Intake of LABA, LAMA or ICS was regular in $91.9 \%$ of patients, even higher for cardiovascular and antidiabetes medication but lower for hyperlipidemia and depression/anxiety medication. Regular intake of respiratory medication did not depend on GOLD groups A-D or grades $1-4$, was highest in patients with concomitant cardiovascular disorders and was lowest for concomitant asthma. It was slightly larger for LAMA and LABA administered via combined compared to single inhalers, and lower when similar compounds were prescribed twice. Most differences did not reach statistical significance owing to the overall high adherence.

Conclusion: Our results indicate a high adherence to respiratory medication in participants of a COPD cohort, especially in those with cardiovascular comorbidities. Compared to the lower adherence reported in the literature for COPD patients, our observations still suggest some room for improvement, possibly through disease management programs.

Keywords: COPD, treatment adherence, respiratory medication, nonrespiratory medication

\section{Introduction}

COPD is a prevalent disorder ${ }^{1}$ associated with significant costs. ${ }^{2}$ Of importance is not only the lung disease but also the high prevalence of, particularly cardiovascular, comorbidities. ${ }^{3,4}$ The comorbidities often result in a high number of prescribed medications, even in relatively mild stages of the lung disease, a fact which is of concern regarding multimedication ${ }^{5}$ but also raises the issue of medication adherence.

Several studies addressed this question via established questionnaires on medication intake and found associations between adherence to COPD medication and patients' characteristics such as quality of life and depression. ${ }^{6-8}$ A comparison of COPD with other chronic diseases is of particular interest ${ }^{9,10}$ to investigate factors 
modulating adherence. For example, in insulin-dependent diabetes, adherence is likely to be higher than in disorders in which nonintake does not have immediate consequences; where COPD is located within this range is not known. A wide variety of inhaled single and combined formulations containing long-acting $\beta$-agonists (LABA), long-acting anticholinergics (LAMA), or inhaled corticosteroids (ICS) are prescribed across all COPD stages, ${ }^{11}$ but most studies on adherence addressed the intake in a more general manner, for example via the Morisky Medication Adherence Scale (MMAS-8), ${ }^{12,13}$ and not on the level of each single prescribed drug.

In view of the frequent comorbidities of COPD, the economic impact of nonadherence ${ }^{14}$ and the potential for adverse effects, ${ }^{5}$ a direct comparison between the adherence to respiratory and nonrespiratory medication in the same patients seems of particular interest. For this purpose, a large cohort of wellcharacterized patients such as COSYCONET ${ }^{15}$ is well suited, although the adherence estimates in such a cohort may be higher than in clinical routine. The use of a longitudinal cohort not only has the advantage of clearly characterized patients but opens up the possibility to ask for the whole panel of respiratory and nonrespiratory medication in great detail. For this, we used a specially designed personal questionnaire, without using in parallel one of the conventional, validated tools, relying on the fact that medication prescription in COSYCONET is fairly constant over time. ${ }^{11}$

Within the German COPD cohort COSYCONET (COPD and Systemic Consequences-Comorbidities Network), ${ }^{15}$ we designed a substudy addressing the intake of respiratory versus nonrespiratory medication and its dependence on patients' characteristics. For this purpose, we used a questionnaire in which for each patient all individual drugs were listed, with the possibility to add newly prescribed drugs. In the case of irregular or nonintake, the reasons for this were assessed. Patients were ensured by a personal letter that the information supplied would be available only to the coordinating center of COSYCONET in pseudonymized form.

\section{Materials And Methods}

\section{Study Population}

All patients from 23 study centers, who were participants of COSYCONET ${ }^{15}$ in the second half of 2017, were contacted per mail and asked for participation. From the patients' last visit within the follow-up, the latest information on medication was taken, and patients received a detailed questionnaire in which their individual medication was listed together with questions regarding their adherence to each of the drugs (see supplement). The first questionnaires were sent out in September 2017 the last ones in March 2018. As the recruitment of COSYCONET was completed at the end of $2013,{ }^{15}$ the last available follow-up visit of patients varied between visit 3 and visit 6 . The questionnaire was accompanied by a letter, in which the patients were ensured that all information provided by them was collected in the coordinating center in Marburg and not available to the study nurses or treating doctors. We considered this as important based on the clinical experience that at least some patients might be reluctant to reveal their true adherence behavior to the medical staff. Ethical approval for the study was approved in addition to that for COSYCONET.

\section{Characterization Of Patients}

For the present analysis, data on medication, the presence of comorbidities, and the COPD categories according to GOLD grades $1-4^{1}$ and GOLD groups A-D ${ }^{1}$ were used. To get an overview, all respiratory medications were recorded (Figure 1), but in subsequent analyses, only LABA, LAMA and ICS were considered. The respective compounds and those referring to comorbidities are listed in Table S1. The statistical evaluation was restricted to the analysis of contingency tables by chi-squared tests, in addition to descriptive figures and tables, assuming significance for $\mathrm{p}<0.05$. Details of the assessments and evaluations are given in the supplement.

\section{Results \\ Study Population}

Overall, 1103 patients from 23 study centers were contacted. Data from 707 responders (64.1\%) of GOLD grades 1-4 were analyzed, comprising 435/272 males/ females, 65/327/244/72 patients of grades $1 / 2 / 3 / 4,{ }^{1}$ and 91/396/11/209 patients of the GOLD groups A/B/C/D. ${ }^{1}$ Patients' characteristics and the distribution of major comorbidities are given in Tables 1 and 2.

\section{Patterns Of Adherence}

\section{Top 25 Of Respiratory Medication}

As an overview of the specific compounds of respiratory medication including short-acting bronchodilators, Figure 1 shows the top 25 prescribed medications, in terms of percentages of prescription, regular, irregular and nonintake. All 


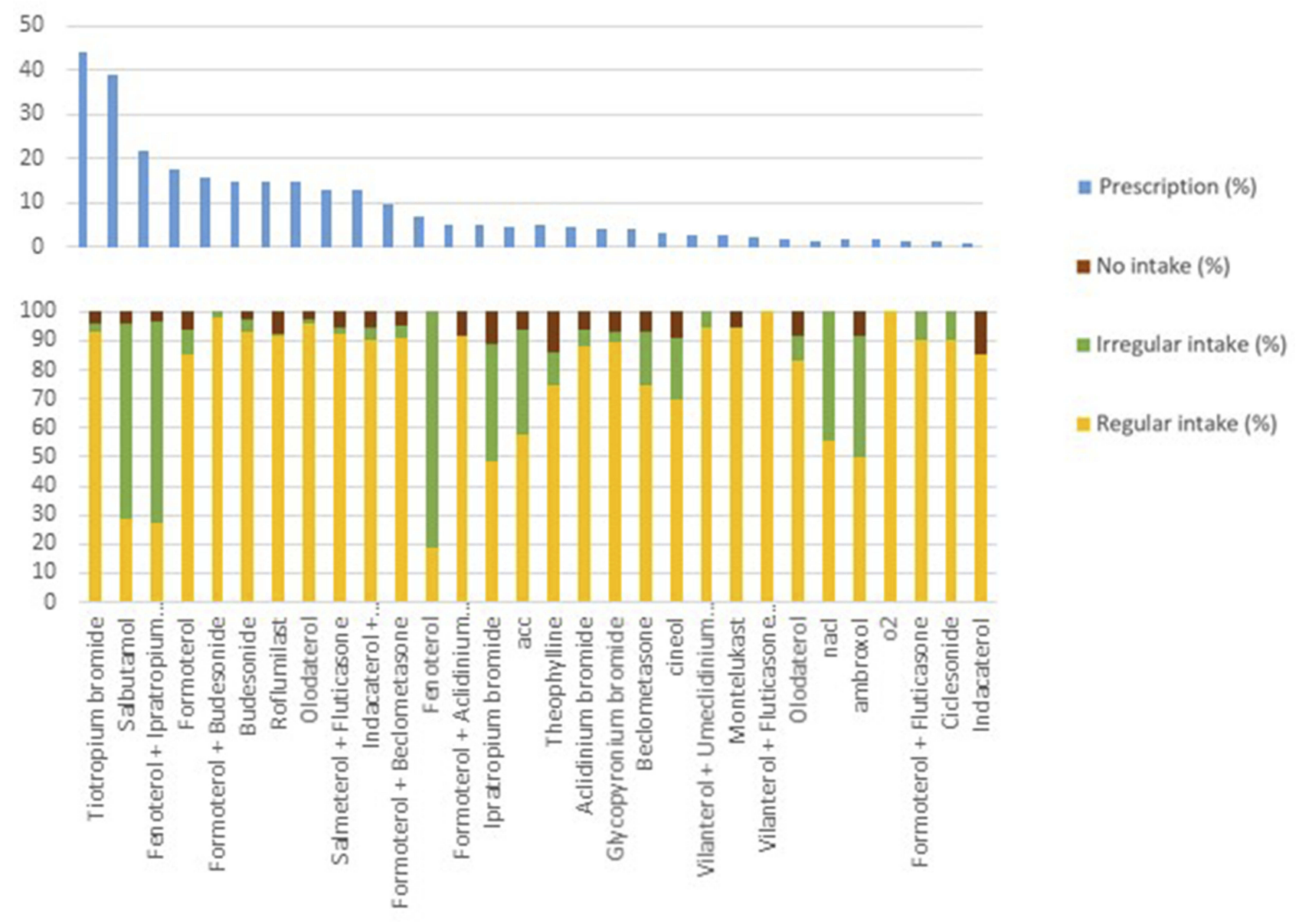

Figure I Top 25 respiratory medications prescribed. The figure shows the top 25 respiratory medications prescribed, most of them inhaled except for montelukast as oral medication, in terms of percentages of prescription, regular, irregular and non-intake. All percentages refer to patients in whom the respective medication was still prescribed.

percentages given below refer to patients in whom the respective medication was still prescribed. Tiotropiumbromide, a long-acting muscarinic agonist (LAMA), and salbutamol, a short-acting $ß$-agonist (SABA), were the most prescribed drugs $(44.3 \%$ and $38.9 \%$ of patients). Overall, LAMA was prescribed in $86.0 \%$ of patients, LABA in $89.5 \%$, and ICS in $60.8 \%$, irrespective of the formulation. Apparently, short-acting compounds were taken predominantly in a nonregular manner, whereas preparations based on long-acting compounds were taken more regularly. Overall, the reported rates of nonintake were low. Overall, the distribution of respiratory medication appeared to reflect the availability of well-introduced and more recent drugs at the time of the study; thus, the most recent triple formulation was found in only two patients.

\section{Comparison Between Diseases}

Figure 2A shows the percentages of prescription of medication for COPD and comorbidities, Figure $2 \mathrm{~B}$ regular intake, Figure 2C irregular intake and Figure 2D no intake; for numerical data see Table S2. In the definition of medication groups (Table S1), respiratory medication included LABA, LAMA and ICS only, excluding shortacting bronchodilators, theophylline and other oral drugs. Diabetes medication was divided into either insulin-type drugs or other antidiabetes drugs. Overall, adherences in terms of regular intake were high, especially for cardiovascular medication (94.6\%), insulin-type drugs (100\%) and antidiabetics excluding insulin-type drugs (93.6\%). There was lower adherence (85.1\%) for psychological medication which also showed a high percentage of nonintake, as well as hyperlipidemia (88.5\%). None of these differences, however, achieved statistical significance.

\section{Dependence On Gender, GOLD Grades And GOLD Groups}

The adherence to respiratory medication comprising LABA, LAMA and ICS (see Table S1) showed slight differences 
Table I Baseline Characteristics Of The Study Cohort ( $n=707)$ According To GOLD Groups A-D (Based On CAT) And GOLD Grades I-4

\begin{tabular}{|c|c|c|c|c|c|c|c|c|c|}
\hline Parameters & $\begin{array}{l}\text { All } \\
n=707\end{array}$ & $\begin{array}{l}\text { GOLD A } \\
n=91\end{array}$ & $\begin{array}{l}\text { GOLD B } \\
n=396\end{array}$ & $\begin{array}{l}\text { GOLD C } \\
n=1 \mid\end{array}$ & $\begin{array}{l}\text { GOLD D } \\
\mathrm{n}=209\end{array}$ & $\begin{array}{l}\text { GOLD I } \\
n=65\end{array}$ & $\begin{array}{l}\text { GOLD } 2 \\
n=327\end{array}$ & $\begin{array}{l}\text { GOLD3 } \\
n=243\end{array}$ & $\begin{array}{l}\text { GOLD } 4 \\
n=72\end{array}$ \\
\hline Gender $\mathrm{m} / \mathrm{f}$ & $435 / 272$ & $56 / 35$ & $251 / / 45$ & $5 / 6$ & $123 / 86$ & $37 / 28$ & $206 / 121$ & $154 / 89$ & $38 / 34$ \\
\hline (\%) & $61.5 / 38.5$ & $61.5 / 38.5$ & $63.4 / 36.6$ & $45.5 / 54.5$ & $58.9 / 41.1$ & $56.9 / 43.0$ & $63.0 / 37.0$ & $63.4 / 36.6$ & $52.8 / 47.2$ \\
\hline Age (years) & $69.0( \pm 8.0)$ & $68.6( \pm 9.0)$ & $69.4( \pm 7.7)$ & $67.6( \pm 9.5)$ & $67.6( \pm 7.8)$ & $70.2( \pm 8.5)$ & $69.6( \pm 8.1)$ & $68.5( \pm 7.4)$ & $64.8( \pm 7.7)$ \\
\hline $\begin{array}{l}\text { BMI }\left(\mathrm{kg} / \mathrm{m}^{2}\right) \\
\text { Smoking status }\end{array}$ & $26.7( \pm 5.2)$ & $26.6( \pm 6.1)$ & $26.8( \pm 4.9)$ & $24.0( \pm 3.1)$ & $26.5( \pm 5.3)$ & $26.6( \pm 4.8)$ & $27.9( \pm 5.4)$ & $25.8( \pm 4.6)$ & $24.0( \pm 4.6)$ \\
\hline $\begin{array}{l}\text { Regular } \\
\text { smoker }\end{array}$ & 147 & 17 & 85 & 3 & 42 & 20 & 77 & 43 & 7 \\
\hline $\begin{array}{l}\text { Occasional } \\
\text { smoker }\end{array}$ & 16 & 2 & 9 & 0 & 5 & 0 & 5 & 9 & 2 \\
\hline Ex-smoker & 499 & 64 & 280 & 7 & 148 & 41 & 221 & 178 & 5 \\
\hline Never-smoker & 45 & 8 & 22 & 1 & 14 & 4 & 24 & 13 & 4 \\
\hline $\begin{array}{l}\mathrm{FEV}, \% \\
\text { predicted }\end{array}$ & $53.7( \pm \mid 8.7)$ & $60.2( \pm 17.7)$ & $55.2( \pm \mid 8.4)$ & $65.6( \pm 17.8)$ & $47.5( \pm 17.8)$ & $89.1( \pm 7.3)$ & $63.0( \pm 8.4)$ & $40.3( \pm 5.5)$ & $25.2( \pm 4.1)$ \\
\hline $\mathrm{FEV}_{\mathrm{l}} / \mathrm{FVC}$ & $0.5 I( \pm 0.1 I)$ & $0.54( \pm 0.11)$ & $0.52( \pm 0.11)$ & $0.56( \pm 0.07)$ & $0.49( \pm 0.11)$ & $0.63( \pm 0.05)$ & $0.57( \pm 0.08)$ & $0.45( \pm 0.09)$ & $0.38( \pm 0.08)$ \\
\hline $\begin{array}{l}\mathrm{FVC} \% \\
\text { predicted }\end{array}$ & $79.8( \pm \mid 9.2)$ & $85.6( \pm 15.8)$ & $80.7( \pm 19.1)$ & $90.6( \pm \mid 8.8)$ & $74.9( \pm 19.7)$ & $108.4( \pm 10.9)$ & $86.4( \pm \mid 3.2)$ & $71.2( \pm \mid 4.2)$ & $53.1( \pm \mid 2.5)$ \\
\hline
\end{tabular}

Note: The table shows mean values $( \pm S D)$ for gender absolute numbers (percentages).

Abbreviations: BMI, body mass index; $\mathrm{FEV}_{1}$, forced expiratory volume in I s; FVC, forced vital capacity; FEV / FVC, ratio of FEV , and FVC.

Table 2 Distribution Of Frequent Comorbidities Of The Study Cohort According To Gender

\begin{tabular}{|l|l|l|l|}
\hline Comorbidities & All & Male & Female \\
\hline Cardiovascular disorders (\%) & 60.0 & 64.8 & 52.2 \\
Hyperlipidemia (\%) & 43.1 & 45.3 & 39.7 \\
Diabetes (\%) & 11.0 & 13.8 & 6.6 \\
Mental disorder (\%) & 25.0 & 20.7 & 32.0 \\
Asthma (\%) & 20.7 & 15.9 & 28.3 \\
\hline
\end{tabular}

Note: The table shows the percentages of the different comorbidities according to gender.

between men and women; in women, regular intake was $90.6 \%$ on average, in men, it was $92.8 \%(p=0.025)$. These data are also given in Table S2, together with the corresponding data for nonrespiratory medication. We next addressed the question whether the GOLD grades or groups were associated with the adherence to respiratory medication. As illustrated in Figure 3, there was only a tendency toward more regular intake in patients of higher GOLD grades. The difference between groups $1-4$ was borderline nonsignificant $(\mathrm{p}=0.050)$. Regarding GOLD groups A-D, there was no significant dependence of regular intake on these $(p>0.05)$, whereby it has to be kept in mind that the number of patients in GOLD group C was low ( $\mathrm{n}=11)$ (see Figure $\mathrm{S} 1$ ).

Associations With Comorbidities And Quality Of Life In the next step, the relationship between the intake of respiratory drugs and the presence of comorbidities was assessed. In addition to the comorbidities such as cardiovascular disease, hyperlipidemia, diabetes and mental disorders, we included the diagnosis of asthma, which was similarly defined as the other comorbidities on the basis of patients' reports and/or disease-specific medication. ${ }^{16}$ Figure 4 shows the intake of respiratory medication across comorbidities, illustrating that there were at most tendencies toward better adherence to respiratory medication in patients with cardiovascular diseases, while adherence was lower in patients with asthma or mental disorders. These differences were not statistically significant $(\mathrm{p}>0.05)$.

At all study visits of COSYCONET, the PhQ-9 test on depression was performed. ${ }^{15}$ When plotting the pattern of intake (Table S1) against the test results categorized according to standard criteria, ${ }^{17}$ there was no significant $(\mathrm{p}>0.05)$ shift toward reduced regular intake in patients with major depression syndrome in comparison with patients with no depression syndrome at all (Figure S2). Similarly, when using the VAS of the EQ-5D divided into four equal subranges, there was no significant $(\mathrm{p}>0.05)$ dependence on medication adherence from the quality of life (Figure S3). Education was divided into three categories: basic, secondary and higher education. We found no significantly $(p>0.05)$ different rates of adherence to respiratory medication (Table $\mathrm{S} 1$ ) in patients with different education (Figure S4). 


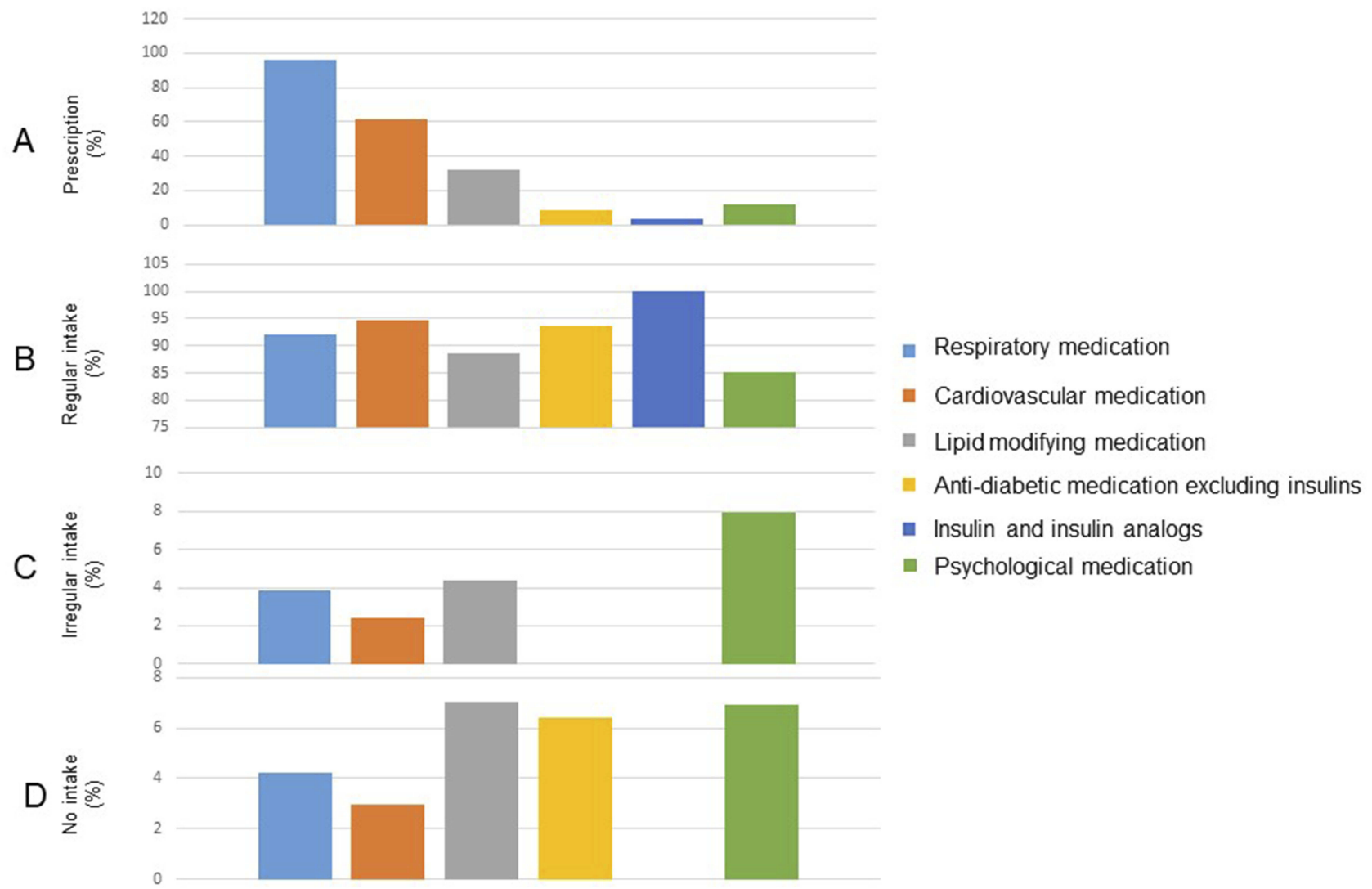

Figure 2 Treatment adherence to different medication groups. The figure shows the percentage of prescription in panel A (uppermost), regular intake in panel B, irregular intake in panel $\mathrm{C}$ and nonintake in panel $\mathrm{D}$. Please note the different scales of the vertical axes, as the scaling has been chosen to provide maximal visual resolution. Medication is grouped into respiratory medication (leftmost), cardiovascular medication, hyperlipidemia-specific medication, antidiabetes medication excluding insulins, insulin-like antidiabetes medication and psychological medication, in that order. For all morbidities, the compounds that were defined as the corresponding medication are listed in Table SI. In case of respiratory medication, this comprised only LAMA, LABA and ICS of any kind, excluding all short-acting bronchodilators and oral medication.

\section{Respiratory Medication: Single Versus Combined Formulations}

A question often discussed is the adherence to combined versus single preparations. To address this issue within our observational study, we selected all patients having both LABA and LAMA as medication and compared the adherence between the following conditions. a) intake of LABA and LAMA in combination, either dual or triple with ICS; b) intake of both LABA and LAMA, each as single formulation, regardless of a potential combination with ICS; we defined the intake of both as regular and the intake of none of both as non-intake; therefore, irregular intake means irregular or nonintake of one of both. Additionally, we analyzed the intake c) of LAMA and d) of LABA separately regardless of the number of formulations containing these drugs; this refers, for example, to patients having LABA+ICS and one LABA additionally. The results are given in Figure 5. Regular intake was slightly higher for the combined formulation of LABA and LAMA, compared to the combination of their single formulations, and was lowest if the condition was met that one or both of the compounds could be given twice, e.g. in combination with ICS. All differences were small and not statistically significant ( $\mathrm{p}>0.05)$.

When all of the previous analyses were performed including the former GOLD 0 patients ${ }^{18}(\mathrm{n}=102$, total $\mathrm{n}=807$ ), there were only minor changes in the percentage values and virtually the same results were obtained.

\section{Reasons For Nonregular Intake Of Respiratory Medication}

Finally, we looked into the reasons for nonregular and nonintake of medication for all respiratory medication listed in Table S1. "Irregular intake" was reported by $3.9 \%$ of all responders $(\mathrm{n}=807)$, whereby $3.0 \%$ reported "intake on demand", while "intake forgotten" and "other reasons" ranged far below $1 \%$. "No intake" was reported by $14.8 \%$ of patients, whereby $10.6 \%$ reported "no more prescribed", $0.8 \%$ were "afraid of side effects", $0.7 \%$ "do not experience effectiveness", $0.3 \%$ reported "in my 


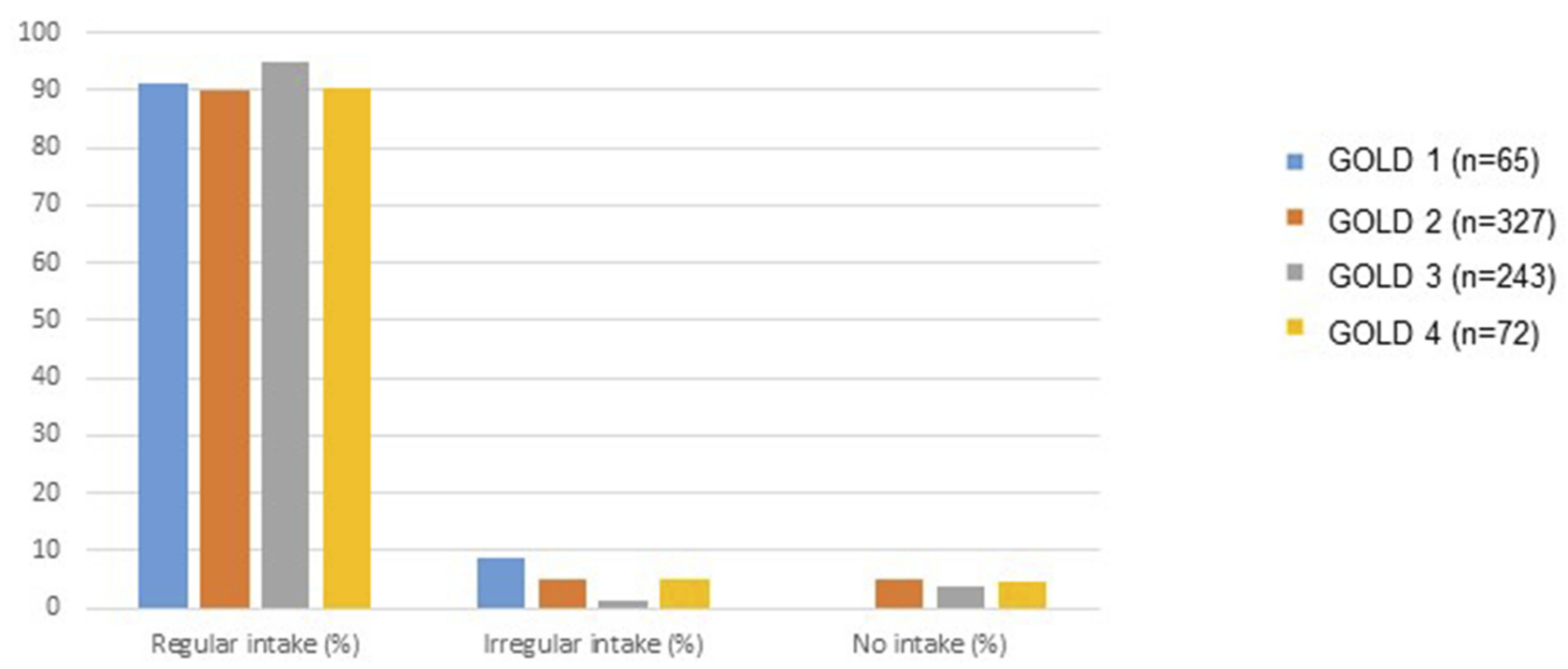

Figure 3 Adherence to respiratory medication according to GOLD grades $0-4$. The figure shows the pattern of medication intake according to GOLD grades $0-4$. The respiratory medication refers to the compounds described in Table SI (LAMA, LABA and ICS). GOLD grade I/2/3/4 n=65/327/243/72. In this graph, regular, irregular and nonintake are arranged along the horizontal axis, and percentages are given on the vertical axis.

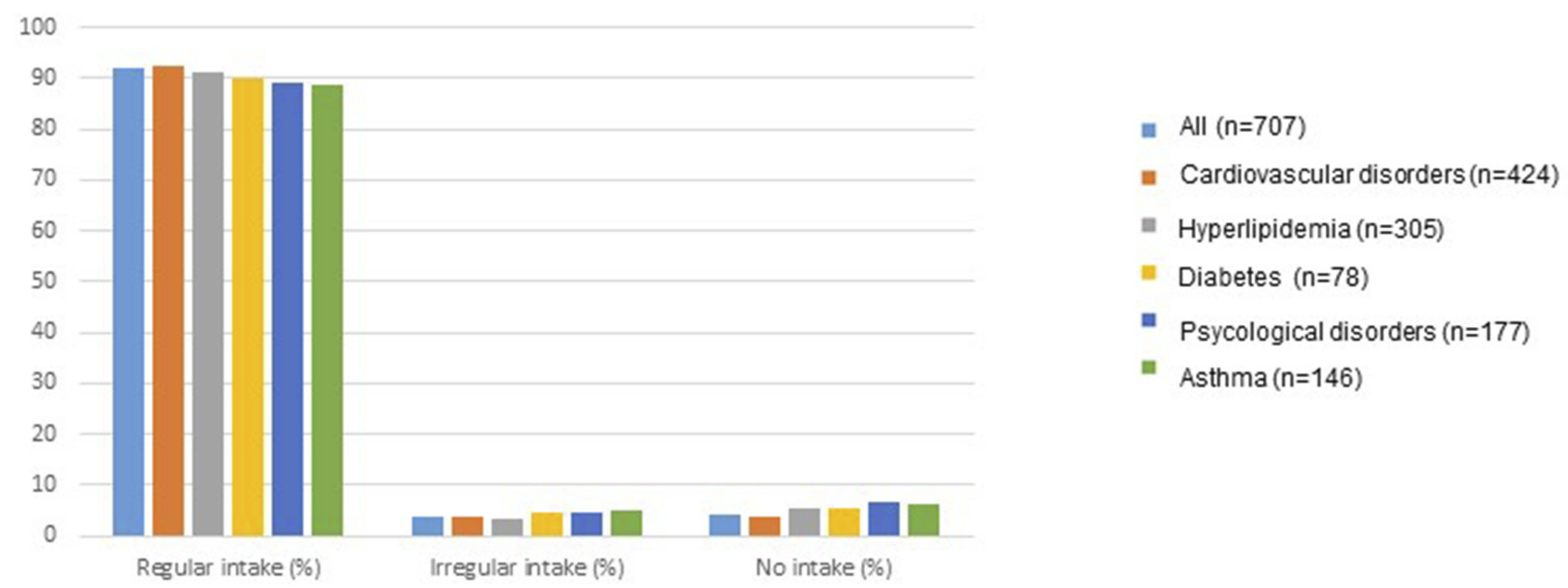

Figure 4 Adherence to respiratory medication in relation to comorbidities. The figure shows the adherence to respiratory medication (see Table SI) in analogy to Figure 3 in relation to comorbidities as defined by patient reports and disease-specific medication (see "Methods"). The vertical axis shows the percentages of patients in the different adherence patterns stratified according to the presence of comorbidities. COPD $n=707$, cardiovascular disorders $n=424$, hyperlipidemia $n=305$, diabetes $n=78$, psychological disease $n=\mid 77$. Additionally, percentages are shown for patients with an additional diagnosis of asthma $n=\mid 46$.

opinion not necessary", and in the remaining patients there was no information.

\section{Discussion}

The present study compared the adherence to COPD medication with that to medication for frequent comorbidities in the same patients of the German COPD cohort COSYCONET. Each patient was asked for each individual medication, and the mode of patients' contact was aiming at a maximum rate of correct answers. The response rate was more than $75 \%$, and reported adherences were very high. About $92 \%$ of patients reported regular intake of long-acting bronchodilators. Regular intake of cardiovascular medication was reported by about $95 \%$ of patients, similar to that of antidiabetes drugs of both insulin- and noninsulin type. Regular intake was lowest for medication against hyperlipidemia or depression/anxiety and in the range of $85-89 \%$, i.e. still high. The adherence to LABA and LAMA showed a tendency toward further improvement for combined formulations. Moreover, the adherence to LABA, LAMA and ICS increased with spirometric GOLD grades, whereas it was independent of GOLD 


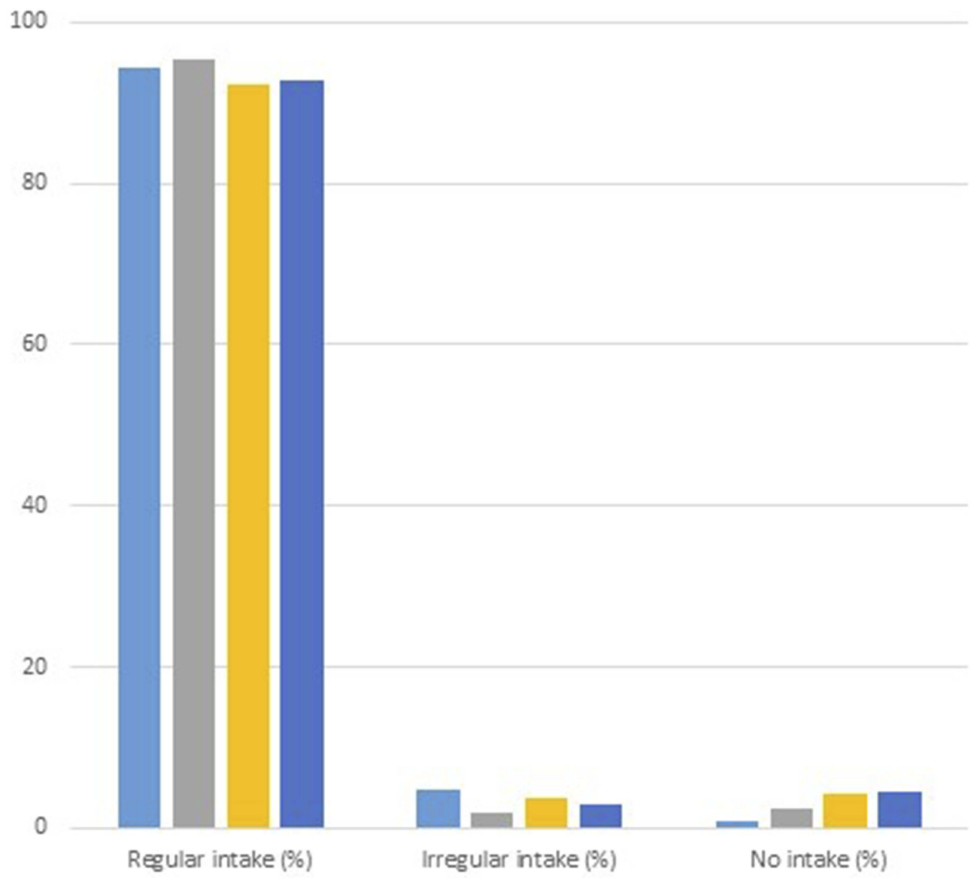

LABA and LAMA single formulations $(n=350)$

LABA and LAMA combined formulation $(\mathrm{n}=197)$

Only LABA (single or in combination) $(n=633)$

Only LAMA (single or in combination) $(n=608)$

Figure 5 Adherence to respiratory medication: single versus combined formulations. The figure shows the pattern of intake of LABA and LAMA, again with adherence pattern along the horizontal axis. The first bar (blue) refers only to patients with exactly one LABA and one LAMA in separate formulations without any other LABA and LAMA. The second bar (gray) refers to patients with exactly one combined formulation of LABA and LAMA without any other prescription of LABA or LAMA. The third (yellow) and fourth bars (dark blue) refer to patients having prescribed any LABA or any LAMA, respectively, even if present in more than one inhaler containing each of these drugs.

groups A-D. There were tendencies toward associations with the level of education and generic quality of life. The fact that medication adherence was high probably resulted in a ceiling effect, which led to the abolishment of some of the influencing factors reported in the literature.

Our study population showed a wide spectrum of medication, particularly respiratory drugs. The spectrum of compounds and formulations as shown in Figure 1 probably reflected the prescription behavior in Germany within the study period of 6 months; thus, recently introduced drugs including inhaled triple formulations but also longestablished drugs were present. For data evaluation, we restricted the panel of drugs to LABA, LAMA and ICS to avoid additional complexity from other, specifically oral drugs such as theophylline and to improve the comparability with the international prescription practice.

Previous studies regarding adherence to respiratory medication in COPD reported rates of regular intake between $29 \%$ and $58 \%{ }^{6,7,19}$ Compared to that, adherence to treatment appeared to be higher in patients with cardiovascular diseases, ranging between $75 \%$ and $80 \%{ }^{20}$ Greater variation of adherence was observed in diabetes, with values between $38 \%$ and $93 \% .^{21,22}$ In our analysis performed in the same patients, adherence to respiratory medication was markedly higher than previously reported ${ }^{6-8,23}$ but still lower than the adherence to cardiovascular medication, which was also high compared to literature data.

At the first view, the difference between respiratory and cardiovascular medication, although small, is surprising, because the intake of many cardiovascular drugs is associated with less immediate benefits, in contrast to respiratory medication. In case of diabetes, the high degree of adherence is probably related to immediate effects, and the fact that no patients reported no regular intake of insulin probably reflects intake on demand; correspondingly, the rate of nonintake was zero. To which extent the high intake of cardiovascular medication is due to the formulation as oral drug remains to be clarified. Another reason could be a higher intensity of educational programs 
in cardiovascular diseases and diabetes. If this should be true, it would suggest further room for improvement in case of respiratory medication, despite the relatively high adherence in the study population. In accordance with this, patients with cardiovascular comorbidities showed a tendency toward a more regular intake of their respiratory medication.

A previous investigation showed an association between the treatment adherence to respiratory and nonrespiratory medication based on data regarding the type and time of prescription from the files. ${ }^{24}$ Another approach is that followed by us, i.e. to compare the adherence to respiratory medication with that for frequent comorbidities in the same patients directly using a detailed personalized questionnaire, which included the reasons for non-adherence. We also addressed the frequently debated question of whether combined inhaler formulations ensure better adherence than single formulations given in parallel. Although a number of pharmacological studies were in favor of this assumption, sometimes the suspicion is raised that this could be partially due to the design of the interventional trials. ${ }^{25,26}$ In our observational study, we observed a similar difference, which was, however, very small due to the high overall adherence. Moreover, we observed a tendency that in case of compounds of the same kind given twice or more in different formulations, the average adherence slightly decreased, suggesting that patients did not appreciate this prescription.

The high degree of adherence to respiratory medication was probably a major reason why we did not observe associations with the diagnosis of depression ${ }^{8}$ or the PhQ score as a measure of depression. Interestingly, there was a slight dependence on the educational status, but different from that reported in the literature. ${ }^{19}$ Adherence tended to be lower in basic compared to secondary education, in accordance with data on the association with the socioeconomic status, ${ }^{8,19}$ but it was again lower in patients with higher education. This might reflect an attitude to manage part of their treatment on their own, depending on their daily clinical state. This interpretation is supported by the observation that the comorbidity of asthma tended to be associated with lower adherence; probably the variability of airway obstruction motivates patients to take part in their medication on demand. In accordance with this, patients of the former COPD grade 0 showed by far the lowest rate of regular intake of their respiratory medication, whereas with increasing obstruction the distribution of adherence was more or less flat. Surprisingly, there was no association between the adherence to respiratory medication and the COPD groups A-D, i.e. the combination of exacerbation rate and symptoms. It has been argued that a higher cutoff value of the CAT score might be more appropriate, ${ }^{27}$ but there was also no relationship to the redefined GOLD groups A-D. In a previous study, ${ }^{8}$ a relationship has been found for a higher cutoff value, but in this study adherence was only $49 \%$, offering more room for variation in accordance with symptoms compared to our adherence of more than $90 \%$.

\section{Limitations}

The study is limited by the fact that we relied on the patients' answers, which were not verified by direct observation of their drug intake. In the accompanying letter, each patient was granted that the whole effort depended on giving honest answers and that the answers would not be available to the treating physicians or study nurses, possibly compromising the patient's respect. The high response rate suggested a low bias from nonresponse, and the fact that all patients available at the time of data acquisition received the questionnaire reduced a bias arising from choosing a subpopulation. In addition, the rate of incomplete questionnaires was low, underlining the feasibility of our approach. It might be argued that the high percentage of reported adherence reflected a severe bias of our approach, particularly when comparing to other, much lower rates of adherence. We believe that patients participating in the cohort over at least four visits represented a positive selection, which would explain the high rate of adherence to respiratory medication. This probably did not affect the comparison between respiratory and nonrespiratory medication. In the questionnaire, this medication was listed in random order, thereby minimizing the likelihood of systematic carryover of positive or negative answers. In addition, evaluation of the DemTect questionnaire for the detection of cognitive impairment in the patients participating in this substudy indicated a very low rate of $1.2 \%$ of patients in which such an impairment could be suspected. The direct approach used by us allowed the rating of adherence whereby we took reference to the EMERGE-reporting checklist as far as possible. ${ }^{28}$

It is possible that also for comorbidities a selection of closely monitored patients occurred, which again could explain the high rate of adherence to the respective medication. We do not know to which extent relative adherences for COPD and comorbidities were affected by this. Another limitation of our study is given by its cross-sectional 
character, which did not allow us to assess whether adherence changed over time. Adherence might increase with increasing severity of the disease over time, although our cross-sectional findings regarding the association with GOLD grades and groups would not favor this.

\section{Conclusion}

The results of this analysis were obtained as a cross-sectional substudy of the COPD cohort COSYCONET, using specifically designed questionnaires that addressed each individual medication in each individual patient. Adherence to COPD medication was higher than previously reported, but that to cardiovascular medication was even slightly greater. It should be noted that patients participating in the cohort may represent a positive selection, which would explain the high rate of adherence. Accordingly, also the regular intake of inhaled respiratory medication tended to be highest in patients with cardiovascular comorbidities, while it was lowest in patients with concomitant asthma. It did not show marked relationships to GOLD grades 1-4 or groups A-D. The difference in adherence between respiratory and cardiovascular medication suggests considering improvements, for example by disease management programs for respiratory diseases, similar to the achievements in cardiovascular diseases.

\section{Abbreviations}

LABA, long-acting ß-agonists; LAMA, long-acting anticholinergics; MMAS-8, Morisky Medication Adherence Scale; ICS, inhaled corticosteroids; SABA, short-acting ß-agonist.

\section{Statement Of Ethics}

All assessments were approved by the central (Marburg [Ethikkommission FB Medizin Marburg]) and local (Bad Reichenhall [Ethikkommission bayerische Landesärztekammer]); Berlin (Ethikkommission Ärztekammer Berlin); Bochum (Ethikkommission Medizinische Fakultät der RUB); Borstel (Ethikkommission Universität Lübeck); Coswig (Ethikkommission TU Dresden); Donaustauf (Ethikkommission Universitätsklinikum Regensburg); Essen (Ethikkommission Medizinische Fakultät Duisburg-Essen); Gießen (Ethikkommission Fachbereich Medizin); Greifswald (Ethikkommission Universitätsmedizin Greifswald); Großhansdorf (Ethikkommission Ärztekammer Schleswig-Holstein); Hamburg (Ethikkommission Ärztekammer Hamburg); MHH Hannover/Coppenbrügge (MHH Ethikkommission);
Heidelberg Thorax/Uniklinik (Ethikkommission Universität Heidelberg); Homburg (Ethikkommission Saarbrücken); Immenhausen (Ethikkommission Landesärztekammer Hessen); Kiel (Ethikkommission Christian-Albrechts-Universität zu Kiel); Leipzig (Ethikkommission Universität Leipzig); Löwenstein (Ethikkommission Landesärztekammer Baden-Württemberg); Mainz (Ethikkommission Landesärztekammer Rheinland-Pfalz); München LMU/Gauting (Ethikkommission Klinikum Universität München); Nürnberg (Ethikkommission Friedrich-Alexander-Universität Erlangen Nürnberg); Rostock (Ethikkommission Universität Rostock); Berchtesgadener Land (Ethikkommission Land Salzburg); Schmallenberg (Ethikkommission Ärztekammer Westfalen-Lippe); Solingen (Ethikkommission Universität Witten-Herdecke); Ulm (Ethikkommission Universität Ulm); Würzburg (Ethikkommission Universität Würzburg) ethical committees and written informed consent was obtained from all patients. The study was conducted from September 2011 to December 2013 and comprised 151 patients recruited within the COSYCONET framework (ClinicalTrials.gov, Identifier: NCT01245933).

For further information see:

Karch A, Vogelmeier C, Welte T, Bals R, Kauczor HU, Biederer J, Heinrich J, Schulz H, Glaser S, Holle R et al: The German COPD cohort COSYCONET: Aims, methods and descriptive analysis of the study population at baseline. Respir Med. 2016;114:27-37.

\section{Acknowledgments}

We would like to thank all patients for their kind participation and Professor Rudolf Maria Huber, University Hospital of Munich, LMU Munich, for helpful suggestions regarding the data evaluation. We also thank all study nurses for their efforts in the distribution of the questionnaires. Names of participating study nurses: Doris Lehnert, Evangelische Lungenklinik Berlin; Birte Struck, Bergmannsheil Berufsgenossenschaftliches Universitätsklinikum Bochum; Lenka Krabbe, Medizinische-Klinik Borstel; Barbara Arikan, Julia Tobias, Klinik Donaustauf; Kornelia Speth, Universitätsklinikum Gießen; Jeanette Pieper, Universitätsmedizin Greifswald; Margret Gleiniger, Britta Markworth, Zaklina Hinz, Pneumologisches Forschungsinstitut Großhansdorf; Ellen Burmann, Hamburger Institut für Therapieforschung Hamburg; Katrin Wons, Medizinische Hochschule Hannover; Ulrike Rieber, 
Beate Schaufler, Thoraxklinik am Universitätsklinikum Heidelberg; Katrin Schwedler, Lungenfachklinik Immenhausen; Sabine Michalewski, Sonja Rohweder, Universitätsklinikum Schleswig-Holstein, Campus Kiel; Patricia Berger, Universitätsklinikum Leipzig; Diana Schottel, Krankenhaus Lindenbrunn, Coppenbrügge; Vivien Janke, Universitätsklinikum Marburg; Rosalie Untsch, Asklepios Fachkliniken, München-Gauting; Jana Graf, Klinikum der Universität München; Anita Reichel, Klinikum Nürnberg; Gertraud Weiß, Erich Traugott, Schön Klinik Berchtesgadener Land; Ilona Kietzmann, Wissenschaftliches Institut Bethanien für Pneumologie e. V, Solingen; Michaela Schrade-Illmann, Beate Polte, Universitätsklinikum-Ulm; Gudrun Hübner, Klinikum Würzburg Mitte gGmbH, Standort Missioklinik, Würzburg. The abstract of this paper was presented at the Congress of the German Society of Pneumology 2019 as oral presentation with interim findings. Furthermore, the abstract of this paper was presented at the 125th Congress of German Society of Internal Medicine as oral presentation with interim findings. The poster's abstract of this Congress was published in "Poster Abstracts" in Der Internist April 2019, Volume 60, Supplement, pp. 1-69.

\section{Author Contributions}

All authors contributed to data analysis, drafting or revising the article, gave final approval of the version to be published and agree to be accountable for all aspects of the work.

\section{Funding}

This work was supported by BMBF Competence Network Asthma and COPD (ASCONET) and performed in collaboration with the German Center for Lung Research (DZL). The project is funded by the German Federal Ministry of Education and Research (BMBF) with grant number 01 GI 0881; and is funded by unrestricted grants from AstraZeneca $\mathrm{GmbH}$, Bayer Schering Pharma AG, Boehringer Ingelheim Pharma GmbH \& Co. KG, Chiesi $\mathrm{GmbH}$, GlaxoSmithKline, Grifols Deutschland $\mathrm{GmbH}$, MSD Sharp \& Dohme GmbH, Mundipharma GmbH, Novartis Deutschland GmbH, Pfizer Pharma GmbH, Takeda Pharma Vertrieb $\mathrm{GmbH} \& \mathrm{Co}$. KG for patient investigations and laboratory measurements.

\section{Disclosure}

Joachim Ficker reports grants from the COSYCONET study supported by Bundesministerium fuer Bildung und
Forschung BMBF, during the conduct of the study. He also received personal fees and nonfinancial support from AstraZeneca, Boehringer Ingelheim, GlaxoSmithKline, Grifols and Novartis, grants, personal fees and nonfinancial support from CSL Behring, outside the submitted work. Claus Vogelmeier reports personal fees from Almirall, grants and personal fees from AstraZeneca, Boehringer Ingelheim, Chiesi, GlaxoSmithKline, Grifols, Mundipharma, Novartis and Takeda, personal fees from Cipla, Berlin Chemie/Menarini, CSL Behring and Teva, grants from German Federal Ministry of Education and Research (BMBF) Competence Network Asthma and COPD (ASCONET), Bayer Schering Pharma AG, MSD and Pfizer, outside the submitted work. Robert Bals reports grants and personal fees from AstraZeneca, Novartis and Boehringer Ingelheim, personal fees from GlaxoSmithKline and Grifols and CSL Behring, grants from German Federal Ministry of Education and Research (BMBF) Competence Network Asthma and COPD (ASCONET), Sander Stiftung, Schwiete Stiftung and Krebshilfe from Mukoviszidose eV, outside the submitted work. Peter Alter reports grants from German Federal Ministry of Education and Research (BMBF) Competence Network Asthma and COPD (ASCONET), and AstraZeneca $\mathrm{GmbH}$, grants and nonfinancial support from Bayer Schering Pharma AG, grants, personal fees and nonfinancial support from Boehringer Ingelheim Pharma GmbH \& Co. KG, grants and nonfinancial support from Chiesi GmbH, grants from GlaxoSmithKline, Grifols Deutschland GmbH, and MSD Sharp \& Dohme GmbH, grants and personal fees from Mundipharma $\mathrm{GmbH}$, grants, personal fees and nonfinancial support from Novartis Deutschland GmbH, grants from Pfizer Pharma GmbH and Takeda Pharma Vertrieb GmbH \& Co. KG, outside the submitted work. Johanna Lutter reports grants from Federal Ministry of Education and Research (grant number 01GI0881), during the conduct of the study. Tobias Welte reports grants from Boehringer, GSK, Novartis, AstraZeneca, during the conduct of the study; personal fees from AstraZeneca, Boehringer, GSK, Novartis, outside the submitted work. The authors report no other conflicts of interest in this work.

\section{References}

1. Vogelmeier CF, Criner GJ, Martinez FJ, et al. Global strategy for the diagnosis, management, and prevention of chronic obstructive lung disease 2017 report: GOLD executive summary. Eur Respir J. 2017;49. doi:10.1183/13993003.00214-2017 
2. Wacker ME, Jörres RA, Schulz H, et al. Direct and indirect costs of COPD and its comorbidities: results from the German COSYCONET study. Respir Med. 2016;111(39-46). doi:10.1016/ j.rmed.2015.12.001

3. Divo M, Cote C, de Torres JP, et al. Comorbidities and risk of mortality in patients with chronic obstructive pulmonary disease. Am J Respir Crit Care Med. 2012;186:155-161. doi:10.1164/rccm. 201201-0034OCrcem.201201-0034OC

4. Wacker ME, Jörres RA, Karch A, et al. Relative impact of COPD and comorbidities on generic health-related quality of life: a pooled analysis of the COSYCONET patient cohort and control subjects from the KORA and SHIP studies. Respir Res. 2016;17(81). doi:10.1186/s12931-016-0401-0

5. Graf J, Lucke T, Herrera R, et al. Compatibility of medication with PRISCUS criteria and identification of drug interactions in a large cohort of patients with COPD. Pulm Pharmacol Ther. 2018;49(123129). doi:10.1016/j.pupt.2018.01.011

6. Ingebrigtsen TS, Marott JL, Nordestgaard BG, et al. Low use and adherence to maintenance medication in chronic obstructive pulmonary disease in the general population. J Gen Intern Med. 2015;30:5159. doi:10.1007/s11606-014-3029-0

7. Agh T, Inotai A, Meszaros A. Factors associated with medication adherence in patients with chronic obstructive pulmonary disease. Respiration. 2011;82:328-334. doi:10.1159/000324453

8. Kokturk N, Polatli M, Oguzulgen IK, et al. Adherence to COPD treatment in Turkey and Saudi Arabia: results of the ADCARE study. Int J Chron Obstruct Pulmon Dis. 2018;13:1377-1388. doi:10.2147/COPD.S150411

9. Briesacher BA, Andrade SE, Fouayzi H, Chan KA. Comparison of drug adherence rates among patients with seven different medical conditions. Pharmacotherapy. 2008;28:437-443. doi:10.1592/phco.28.4.437

10. Napolitano F, Napolitano P, Angelillo IF, Collaborative Working G. Medication adherence among patients with chronic conditions in Italy. Eur J Public Health. 2016;26:48-52. doi:10.1093/eurpub/ckv147

11. Graf Jana JAR, Tanja L, Nowak Dennis VF. Claus. Medikamentöse Therapie der COPD. Deutsches Ärzteblatt. 2018;37:599-605.

12. Morisky DE, Ang A, Krousel-Wood M, Ward HJ. Predictive validity of a medication adherence measure in an outpatient setting. $J$ Clin Hypertens (Greenwich). 2008;10:348-354.

13. Montes de Oca M, Menezes A, Wehrmeister FC, et al. Adherence to inhaled therapies of COPD patients from seven Latin American countries: the LASSYC study. PLoS One. 2017;12:e186777. doi:10.1371/journal.pone. 0186777

14. Dekhuijzen R, Lavorini F, Usmani OS, van Boven JFM. Addressing the impact and unmet needs of nonadherence in asthma and chronic obstructive pulmonary disease: where do we go from here? J Allergy Clin Immunol Pract. 2018;6:785-793. doi:10.1016/j.jaip.2017.11.027

15. Karch A, Vogelmeier C, Welte T, et al. The German COPD cohort COSYCONET: aims, methods and descriptive analysis of the study population at baseline. Respir Med. 2016;114:27-37. doi:10.1016/j. rmed.2016.03.008
16. Lucke T, Herrera R, Wacker M, et al. Systematic analysis of selfreported comorbidities in large cohort studies - a novel stepwise approach by evaluation of medication. PLoS One. 2016;11: e0163408. doi:10.1371/journal.pone. 0163408

17. Spitzer D. Marginal medicine. JAMA.1999;281:1082. author reply 1083. doi:10.1001/jama.281.12.1082

18. Pauwels RA, Buist AS, Calverley PM, Jenkins CR, Hurd SS. Global strategy for the diagnosis, management, and prevention of chronic obstructive pulmonary disease. NHLBI/WHO Global Initiative for Chronic Obstructive Lung Disease (GOLD) workshop summary. Am J Respir Crit Care Med. 2001;163:1256-1276. doi:10.1164/ ajrccm.163.5.2101039

19. Krauskopf K, Federman AD, Kale MS, et al. Chronic obstructive pulmonary disease illness and medication beliefs are associated with medication adherence. COPD. 2015;12:151-164. doi:10.3109/15412 555.2014.922067

20. Chowdhury R, Khan H, Heydon E, et al. Adherence to cardiovascular therapy: a meta-analysis of prevalence and clinical consequences. Eur Heart J. 2013;34:2940-2948. doi:10.1093/eurheartj/eht295

21. Krass I, Schieback P, Dhippayom T. Adherence to diabetes medication: a systematic review. Diabet Med. 2015;32:725-737. doi:10. 1111/dme.12651

22. Cramer JA. A systematic review of adherence with medications for diabetes. Diabetes Care. 2004;27:1218-1224. doi:10.2337/diacare. 27.5.1218

23. Vestbo J, Anderson JA, Calverley PMA, et al. Adherence to inhaled therapy, mortality and hospital admission in COPD. Thorax. 2009;64:939-943. doi:10.1136/thx.2009.113662

24. Dhamane AD, Schwab P, Hopson S, et al. Association between adherence to medications for COPD and medications for other chronic conditions in COPD patients. Int J Chron Obstruct Pulmon Dis. 2017;12:115-122. doi:10.2147/COPD.S114802

25. Tamura G, Ohta K. Adherence to treatment by patients with asthma or COPD: comparison between inhaled drugs and transdermal patch. Respir Med. 2007;101:1895-1902. doi:10.1016/j.rmed. 2007.05.001

26. Bateman ED, Ferguson GT, Barnes N, et al. Dual bronchodilation with QVA149 versus single bronchodilator therapy: the SHINE study. Eur Respir J. 2013;42(1484-1494). doi:10.1183/09031936.00200212

27. Smid DE, Franssen FME, Gonik M, et al. redefining cut-points for high symptom burden of the global initiative for chronic obstructive lung disease classification in 18,577 patients with chronic obstructive pulmonary disease. J Am Med Dir Assoc. 2017;18:1097 e1011-1097 e1024. doi:10.1016/j.jamda.2017.09.003

28. Helmy R, Zullig LL, Dunbar-Jacob J, et al. ESPACOMP Medication Adherence Reporting Guidelines (EMERGE): a reactive-Delphi study protocol. BMJ Open. 2017;7:e13496. doi:10.1136/bmjopen2016-013496
Patient Preference and Adherence

\section{Publish your work in this journal}

Patient Preference and Adherence is an international, peer-reviewed, open access journal that focusing on the growing importance of patient preference and adherence throughout the therapeutic continuum. Patient satisfaction, acceptability, quality of life, compliance, persistence and their role in developing new therapeutic modalities and compounds to optimize clinical outcomes for existing disease states are major areas of interest for the journal. This journal has been accepted for indexing on PubMed Central. The manuscript management system is completely online and includes a very quick and fair peer-review system, which is all easy to use. Visit http:// www.dovepress.com/testimonials.php to read real quotes from published authors. 